\title{
Using perturbation methods and Laplace-Padé approximation to solve nonlinear problems
}

U. Filobello-Nino, H. Vazquez-Leal, Y. Khan, A. Yildirim, V. M. Jimenez-Fernandez, A. L. Herrera-May, R. Castaneda-Sheissa, and J. Cervantes-Perez 


\title{
USING PERTURBATION METHODS AND LAPLACE-PADÉ APPROXIMATION TO SOLVE NONLINEAR PROBLEMS
}

\author{
U. FILOBELLO-NINO, H. VAZQUEZ-LEAL, Y. KHAN, A. YILDIRIM, \\ V. M. JIMENEZ-FERNANDEZ, A. L. HERRERA-MAY, R. CASTANEDA-SHEISSA, \\ AND J. CERVANTES-PEREZ
}

Received 5 April, 2012

\begin{abstract}
In this paper, the perturbation method and Padé transformation are used to provide an approximate solution of elliptic integrals of the second kind and of complete integrals of the first kind. Besides, we used the obtained results to calculate an analytic expression for the period of a simple pendulum. The method has an acceptable accuracy for high values of the initial amplitude, compared to the relative error $<1.7 \%$ for initial angles $\theta \leq 70^{\circ}$.
\end{abstract}

2000 Mathematics Subject Classification: 37M05; 47A55

Keywords: Perturbation method, nonlinear problems, approximation methods

\section{INTRODUCTION}

Among others, the perturbation method is one of the pioneering techniques of approaching various kinds of nonlinear problems. This procedure was introduced by S. D. Poisson and extended by J. H. Poincare. Although the method appeared in the early 19th century, the application of a perturbation procedure to solve nonlinear differential equations was used only a bit later. The most significant efforts were focused on celestial mechanics, fluid mechanics, and aerodynamics [7,19].

In general, it is assumed that the differential equation can be expressed as the sum of two parts: a linear part and a nonlinear one. The nonlinear part is considered as a small perturbation through a small parameter (the perturbation parameter). The assumption that the nonlinear part is small compared to the linear is considered as a disadvantage of the method. There are other modern alternatives to find approximate solutions of the differential equations that describe some nonlinear problems such as those based on variational approaches [3,17], Tanh method [9], Exp-function [24], Adomian's decomposition method [1,4], parameter expansion [25], homotopy perturbation method $[6,8,10,11,13-16,18,22,23]$, homotopy perturbation transform Padé method (HPTPM) [12], homotopy perturbation transform method (HPTM) [20], among others. 
This paper applies the PM method to find an approximation of the value of second kind elliptic integrals; this is done by solving a first order nonlinear differential equation, which depends on one perturbation parameter. We will see that our approximation has good accuracy, even for large values of the perturbation parameter. Finally, we will couple the PM and Padé methods to obtain an approximation of the oscillation period for a nonlinear pendulum.

This paper is organized as follows. In Section 2, a short introduction about the basic elements of elliptic integrals is provided. In Section 3, we introduce the basic idea of the PM method. Section 4 will provide a brief introduction to the Padé approximation. In Section 5, we provide an application of the PM method. Section 6 presents an approximate solution of the complete elliptic integral of the first kind by using Laplace-Padé approximation. In Section 7, we obtain the period for a pendulum in an approximate way. In Section 8, we summarize our findings. Finally, a brief conclusion is given in Section 9.

\section{ELLIPTIC INTEGRALS AND THE PERIOD FOR A SIMPLE PENDULUM}

Elliptic integrals are integrals without closed solution, they have been used extensively for evaluating integrals. For instance, integrals of the form

$$
I=\int_{0}^{t} R\left(x, \sqrt{a x^{4}+b x^{3}+c x^{2}+d x+e}\right) d x,
$$

where $R$ is a rational function of $x$ and the radical; $a, b, c, d$, and $e$ are constants, may be expressed in terms of elliptic integrals [2]. Furthermore, elliptic integrals are important because they are useful to solve physical problems, like the case of the magnetic vector potential for a circular current loop [2] and the orbital equation for a particle subject to certain central potentials [7].

Elliptic integrals are divided in three cases:

(1) Incomplete elliptic integral of the first kind. Which is defined as

$$
K(\epsilon, \phi)=\int_{0}^{\phi} \frac{d \theta}{\sqrt{1-\epsilon^{2} \sin ^{2} \theta}}, \quad 0 \leq \epsilon<1,
$$

where $\phi$ is the amplitude of $K(\epsilon, \phi)$, and $\epsilon$ is their modulus. For $\phi=\frac{\pi}{2}$, we have the complete elliptic integral of the first kind,

$$
K(\epsilon)=\int_{0}^{\pi / 2} \frac{d \theta}{\sqrt{1-\epsilon^{2} \sin ^{2} \theta}}, \quad 0 \leq \epsilon<1 .
$$

(2) Incomplete elliptic integral of the second kind. It is defined as

$$
E(\epsilon, \phi)=\int_{0}^{\phi} \sqrt{1-\epsilon^{2} \sin ^{2} \theta} d \theta, \quad 0 \leq \epsilon<1 .
$$


For $\phi=\frac{\pi}{2}$, we have the complete elliptic integral of the second kind

$$
E(\epsilon)=\int_{0}^{\pi / 2} \sqrt{1-\epsilon^{2} \sin ^{2} \theta} d \theta, \quad 0 \leq \epsilon<1 .
$$

(3) Incomplete elliptic integral of the third kind. It is defined as

$$
\Pi(\epsilon, n, \phi)=\int_{0}^{\phi} \frac{d \theta}{\left(1+n \sin ^{2} \theta\right) \sqrt{1-\epsilon^{2} \sin ^{2} \theta}}, \quad 0 \leq \epsilon<1,
$$

where $n$ is a constant $n \neq 0$, that is, if $n=0$ (2.5) would be identical to (2.1).

Again, for the case $\phi=\frac{\pi}{2}$, the complete elliptic integral of the third kind is obtained

$$
\Pi(\epsilon, n)=\int_{0}^{\pi / 2} \frac{d \theta}{\left(1+n \sin ^{2} \theta\right) \sqrt{1-\epsilon^{2} \sin ^{2} \theta}}, \quad 0 \leq \epsilon<1 .
$$

\section{BASIC IDEA OF THE PERTURBATION METHOD}

Let the differential equation of one-dimensional nonlinear system be in the form

$$
L(x)+\epsilon N(x)=0,
$$

where we assume that $x$ is a function of one variable $x=x(t), L(x)$ is a linear operator which, in general, contains derivatives in terms of $t . N(x)$ is a nonlinear operator, and $\epsilon$ is a small parameter.

By considering the nonlinear term in (3.1) as a perturbation, we assume that solution (3.1) can be written as a power series in the small parameter $\epsilon$

$$
x(t)=x_{0}(t)+\epsilon x_{1}(t)+\epsilon^{2} x_{2}(t)+\epsilon^{3} x_{3}(t)+\cdots .
$$

By substituting (3.2) into (3.1) and equating terms having identical powers of $\epsilon$, we obtain a number of differential equations that can be integrated, recursively, to find the values for the functions $x_{0}(t), x_{1}(t), x_{2}(t), \ldots$

\section{PADÉ APPROXIMANT}

A rational approximation to $f(x)$ on $[a, b]$ is the quotient of two polynomials $P_{N}(x)$ and $Q_{M}(x)$ of degrees $N$ and $M$, respectively. We use the notation $R_{N, M}(x)$ to denote this quotient. The $R_{N, M}(x)$ Padé approximations of a function $f(x)$ are given by $[5,12,21]$

$$
R_{N, M}=\frac{P_{N}(x)}{Q_{M}(x)} \quad \text { for } a \leq x \leq b .
$$

The method of Padé requires that $f(x)$ and its derivative be continuous at $x=0$. The polynomials used in (4.1) are

$$
P_{N}(x)=p_{0}+p_{1} x+p_{2} x^{2}+\cdots+p_{N}(x),
$$




$$
Q_{M}(x)=q_{0}+q_{1} x+q_{2} x^{2}+\cdots+q_{M}(x) .
$$

The polynomials in (4.2) and (4.3) are constructed so that $f(x)$ and $R_{N, M}(x)$ agree at $x=0$ and their derivatives up to degree $N+M$ agree at $x=0$. In the case $Q_{0}(x)=1$, the approximation is just the Maclaurin expansion for $f(x)$. For a fixed value of $N+M$ the error is the smallest when $P_{N}(x)$ and $Q_{M}(x)$ have the same degree or when $P_{N}(x)$ has degree one higher than $Q_{M}(x)$.

Note that the constant coefficient of $Q_{M}$ is $q_{0}-1$. This is permissible, because it can be noted that 0 and $R_{N, M}(x)$ do not change when both $P_{N}(x)$ and $Q_{M}(x)$ are divided by the same constant. Hence, the rational function $R_{N, M}(x)$ has $N+$ $M+1$ unknown coefficients. Assume that $f(x)$ is analytic and it has the Maclaurin expansion

$$
f(x)=a_{0}+a_{1} x+a_{2} x^{2}+\cdots+a_{k} x^{k}+\cdots .
$$

From the difference $f(x) Q_{M}(x)-P_{N}(x)=Z(x)$, we obtain

$$
\left\lceil\sum_{i=0}^{\infty} a_{i} x^{i}\right\rceil\left\lceil\sum_{i=0}^{M} q_{i} x^{i}\right\rceil-\left\lceil\sum_{i=0}^{N} p_{i} x^{i}\right\rceil=\left\lceil\sum_{i=N+M+1}^{\infty} c_{i} x^{i}\right\rceil .
$$

The lower index $j=N+M+1$ in the summation on the right side of (4.5) is chosen because the first $N+M$ derivatives of $f(X)$ and $R_{N, M}(x)$ should converge at $x=0$.

When the left side of (4.5) is multiplied and coefficients with powers of $x^{i}$ are set equal to zero for $k=0,1,2, \ldots, N+M$, the results are a system of $N+M+1$ linear equations

$$
\begin{aligned}
a_{0}-p_{0} & =0, \\
q_{1} a_{0}+a_{1}-p_{1} & =0, \\
q_{2} a_{0}+q_{1} a_{1}+a_{2}-p_{2} & =0, \\
q_{3} a_{0}+q_{2} a_{1}+q_{1} a_{2}+a_{3}-p_{3} & =0, \\
q_{M} a_{N-M}\left|q_{M-1} a_{N-M-1}\right| a_{N}-p_{N} & =0,
\end{aligned}
$$

and

$$
\begin{array}{r}
q_{M} a_{N-M+1}+q_{M-1} a_{N-M+2}+\cdots+q_{1} a_{N}+a_{N+2}=0, \\
q_{M} a_{N-M+2}+q_{M-1} a_{N-M+3}+\cdots+q_{1} a_{N+1}+a_{N+3}=0, \\
\vdots \\
q_{M} a_{N}+q_{M} a_{N+1}+\cdots+q_{1} a_{N+M+1}+a_{N+M}=0 .
\end{array}
$$

Notice that, for each equation, the sum of the subscripts on the factors of each product is the same. This sum increases consecutively from 0 to $N+M$. The $M$ equations in (4.7) involve only the unknowns $q_{1}, q_{2}, \ldots, q_{M}$ and they must be solved first. Then the equations in (4.6) are used successively to find $p_{1}, p_{2}, \ldots, p_{N}[12,21]$. 


\section{Application OF PM METhOd to SOlVE THE COMPLETE Elliptic INTEGRALS OF THE SECOND KIND AND ELLIPTIC INTEGRAL OF THE FIRST} KIND

This section deals with the solution of (2.2), (2.3), and (2.4) by following a procedure used in [22], where (2.3) is represented as follows

$$
y(\phi)=\int_{0}^{\phi} \sqrt{1-\epsilon^{2} \sin ^{2} \theta} d \theta, \quad 0 \leq \phi \leq \pi / 2 .
$$

This integral can be reformulated as a nonlinear differential equation

$$
y^{\prime 2}(\phi)-1+\epsilon^{2} \sin ^{2} \phi=0 .
$$

By evaluating the integral (5.1) at $\phi=0$, the result will be zero. Therefore, the initial condition for the differential equation (5.2) should be $y(0)=0$.

It is possible to find a handy solution for (5.2) by applying the PM method. To apply the method, first, we identify terms

$$
\begin{gathered}
L(\phi)=y^{\prime 2}(\phi)-1, \\
N(\phi)=\sin ^{2} \phi, \quad(\text { see }(4.1)),
\end{gathered}
$$

and $\epsilon^{2}$ with the PM parameter. Notice that, for this case, $N$ is truly linear, showing the flexibility of the method.

By assuming a solution for (5.2), in the form

$$
y(\phi)=v_{0}(\phi)+\eta v_{1}(\phi)+\eta^{2} v_{2}(\phi)+\cdots \quad\left(\eta=\epsilon^{2}\right) \quad(\text { see }(3.2)),
$$

and equating terms with equal powers of $\eta$, it can be solved for $v_{0}(\phi), v_{1}(\phi), v_{2}(\phi), \ldots$, and so on. As a result, a very good approximation is obtained considering terms up to third order.

$$
\begin{aligned}
\left.\eta^{0}\right) & v_{o}^{\prime 2}-1=0 \\
\left.\eta^{1}\right) & =0 \\
\left.\eta^{2}\right) & 2 v_{0}^{\prime} v_{1}^{\prime}+\sin ^{2} \theta=0 \\
\left.\eta^{3}\right) & v_{1}^{\prime 2}+2 v_{0}^{\prime} v_{2}^{\prime}=0 \\
2 v_{0}^{\prime} v_{3}^{\prime}+2 v_{1}^{\prime} v_{2}^{\prime} & =0
\end{aligned}
$$

In order to fulfil the initial condition from (5.2) $(y(0)=0)$, it follows that $v_{0}(0)=$ $0, v_{1}(0)=0, v_{2}(0)=0, v_{3}(0)=0$, and so on. Thus, the results are

$$
\begin{array}{r}
v_{0}(\phi)=\phi, \\
v_{1}(\phi)=-\frac{1}{4} \phi+\frac{1}{8} \sin 2 \phi, \\
v_{2}(\phi)=-\frac{3}{64} \phi+\frac{1}{32} \sin 2 \phi-\frac{1}{256} \sin 4 \phi,
\end{array}
$$




$$
v_{3}(\phi)=-\frac{5}{96}\left[\frac{3}{8} \phi-\frac{1}{4} \sin 2 \phi+\frac{1}{32} \sin 4 \phi\right]+\frac{1}{96} \cos \phi \sin ^{5} \phi,
$$

By substituting (5.10), (5.11), (5.12), and (5.13) into (5.5); and considering that $E(\epsilon, \phi)=y(\phi)$, it follows that an approximate solution to the incomplete second kind elliptic integral would be

$$
\begin{aligned}
E(\eta, \phi)= & \phi+\eta\left[\frac{1}{8} \sin 2 \phi-\frac{1}{4} \phi\right] \\
& +\eta^{2}\left[-\frac{3}{64} \phi+\frac{1}{32} \sin 2 \phi-\frac{1}{256} \sin 4 \phi\right] \\
& +\eta^{3}\left[-\frac{15}{768} \phi+\frac{5}{384} \sin 2 \phi-\frac{5}{3072} \sin 4 \phi+\frac{1}{96} \cos \phi \sin ^{5} \phi\right] \\
& +\cdots
\end{aligned}
$$

For convenience, (5.14) is rewritten in the form

$$
\begin{aligned}
E(\eta, \phi)= & \phi\left[1-\frac{1}{4} \eta-\frac{3}{64} \eta^{2}-\frac{15}{768} \eta^{3}+\cdots\right] \\
& +\sin 2 \phi\left[\frac{1}{8} \eta+\frac{1}{32} \eta^{2}+\left(\frac{5}{384}+\frac{\sin ^{4} \phi}{192}\right) \eta^{3}+\cdots\right] \\
& -\sin 4 \phi\left[\frac{1}{256} \eta^{2}+\frac{5}{3072} \eta^{3}+\cdots\right] \\
& +\cdots
\end{aligned}
$$

Evaluating (5.15) for $\phi=\pi / 2$, it becomes the complete elliptic integral of the second kind (approximation (2.4))

$$
E(\epsilon)=\frac{\pi}{2}\left[1-\frac{1}{4} \epsilon^{2}-\frac{3}{64} \epsilon^{4}-\frac{15}{768} \epsilon^{6}+\cdots\right],
$$

In order to find an approximate solution for integral (2.2), we use the following equation which relates the complete elliptic integrals [2]

$$
K(\epsilon)=E(\epsilon)-\epsilon \frac{d E(\epsilon)}{d \epsilon} .
$$

When substituting (5.16) into (5.17), it is possible to obtain the following expression for $K(\epsilon)$

$$
K(\epsilon)=\frac{\pi}{2}\left[1+\frac{1}{4} \epsilon^{2}+\frac{9}{64} \epsilon^{4}+\frac{15}{153} \epsilon^{6}+\cdots\right] .
$$




\section{AN APPROXIMATE SOLUTION BY USING LAPLACE-PADÉ TRANSFORMATION AND PM METHOD}

In this section we will describe the use of Laplace transform and Padé transformation to deal with the truncated series (5.18) obtained by the PM method in order to improve the approximation and the proposed valid range for the same series [21].

First, Laplace transformation is applied to (5.18) and then $1 / \epsilon$ is written instead of $s$ in the obtained equation. Then, Padé approximant [2/2] is applied and $1 / s$ is written instead of $\epsilon$. Finally, by using the inverse Laplace transformation, we obtain the modified approximate solution

$$
K(\epsilon)=\frac{\pi}{2} \cosh \left(\frac{\sqrt{2}}{2} \epsilon\right) .
$$

\section{CASE Study}

One relevant application to be considered here, for the complete elliptic integral of the first kind (2.2), is the problem of calculating the period of a nonlinear pendulum. The simple pendulum's oscillatory motion is among the most investigated motions in physics; many nonlinear phenomena in several fields of science and technology are governed by pendulum-like differential equations.

For small amplitude oscillations, the approximate period of a simple pendulum is given by

$$
T_{0} \approx 2 \pi \sqrt{\frac{l}{g}},
$$

where $l$ is the length and $g$ is the acceleration of gravity.

The exact value of the period of oscillation is

$$
T=4 \sqrt{\frac{l}{g}} \int_{0}^{\pi / 2} \frac{d \theta}{\sqrt{1-\epsilon^{2} \sin ^{2} \theta}} ; \quad \epsilon=\sin \left(\frac{\theta_{0}}{2}\right),
$$

where $\theta_{0}$ is the initial amplitude. It is clear that we can express (7.1) in terms of the complete integral of the first kind (2.2)

$$
T=4 \sqrt{\frac{l}{g}} K(\epsilon),
$$

our procedure allows obtaining two different approximations to the oscillation period for a pendulum. The first is obtained by substituting (5.18) into (7.2)

$$
T=2 \pi \sqrt{\frac{l}{g}}\left[1+\frac{1}{4} \epsilon^{2}+\frac{9}{64} \epsilon^{4}+\frac{15}{153} \epsilon^{6}+\cdots\right] .
$$




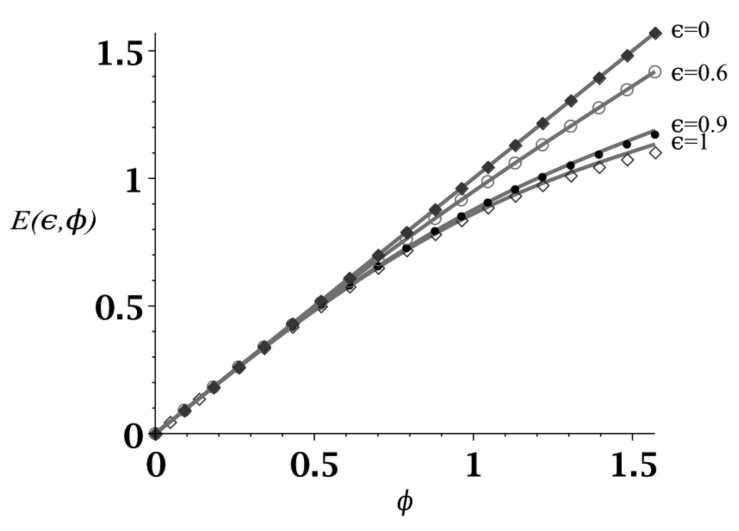

FIGURE 1. Exact numerical solution (solid line); approximate solutions (5.15) for $\epsilon=[0,0.6,0.9,1]$ represented by: solid diamond, circle, solid circle, diamond, respectively.

The second is the result of substituting (6.1) into (7.2), which lead us to

$$
T=2 \pi \sqrt{\frac{l}{g}} \cosh \left(\frac{\sqrt{2}}{2} \epsilon\right) .
$$

For comparison purposes, period (7.2), (7.3) and (7.4) will be normalized to

$$
\hat{T}=\frac{1}{4} \sqrt{\frac{g}{l}} T .
$$

In the next section, we will analyse and compare $\hat{T}$ for (7.2), (7.3) and (7.4).

\section{DISCUSSION}

Nonlinear phenomena appear in broad scientific fields like applied mathematics, physics, and engineering. Scientists in those disciplines constantly face the task of finding solutions of nonlinear ordinary differential equations. The fact that the PM method depends on a parameter which is assumed to be small, suggests that the method has limited range. In this work, the PM method has been applied to the problem of evaluating, approximately, complete or incomplete elliptic integrals of the second kind by using the nonlinear differential equation (5.2). From the obtained results, an approximation was achieved for the complete elliptic integral of the first kind and, as a consequence, for the period of a nonlinear pendulum. We will see that even though the PM does not provide a satisfactory approximation in the latter case, when it is coupled with Padé methods, we may obtain a better approximation. 


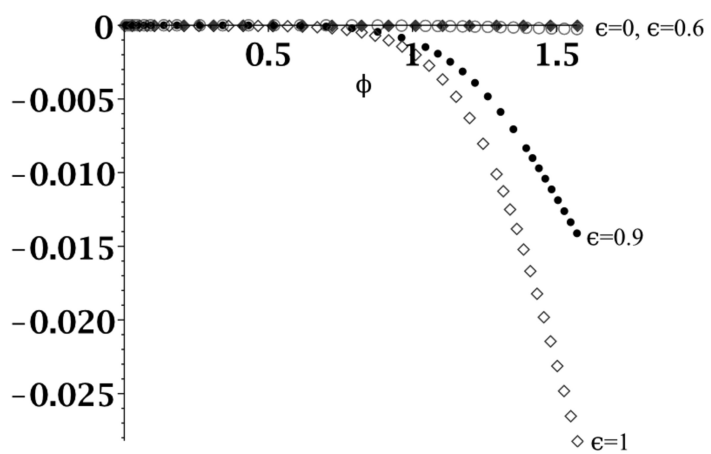

FIGURE 2. Relative error from approximated solutions given in Figure 1 .

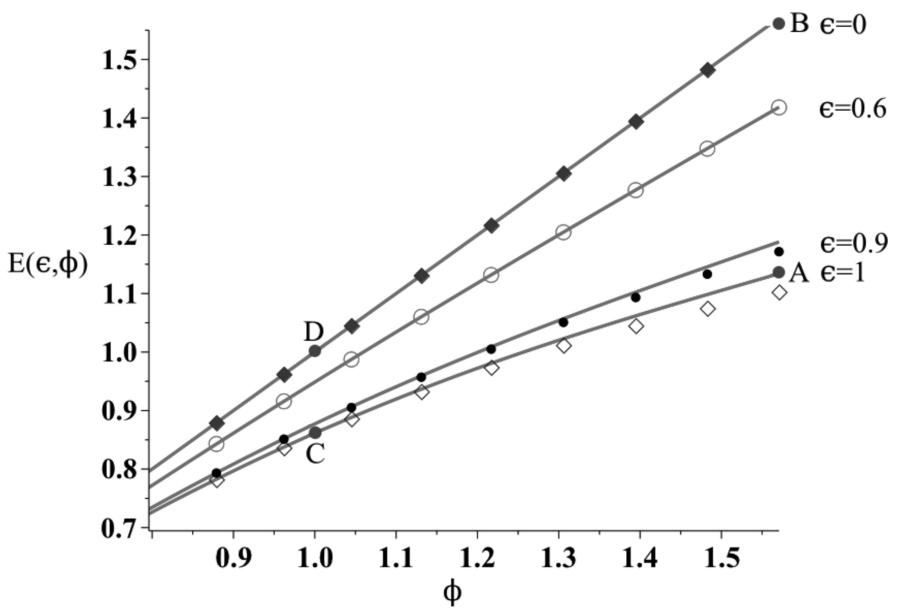

FIGURE 3. Zoom from figure 1.

\subsection{Elliptic integrals of the second kind}

For the case of elliptic integrals of the second kind, we obtained highly accurate approximations for both, complete and incomplete, integrals. From (5.16), for instance, in the case of the complete integral, the limit

$$
\lim _{\epsilon \rightarrow 1} E(\epsilon)=1.073,
$$

means that the error is about $7 \%$. 


\begin{tabular}{cccccc}
\hline$\theta$ & Numerical & PM-Padé & RE\% & PM & RE\% \\
\hline \hline 0 & 1.570796 & 1.570796 & 0 & 1.570796 & 0 \\
4 & 1.571275 & 1.571275 & $1.93 \mathrm{e}-05$ & 1.572716 & -0.0917 \\
8 & 1.572712 & 1.572708 & 0.000308 & 1.578537 & -0.37 \\
10 & 1.573792 & 1.573780 & 0.000754 & 1.582968 & -0.583 \\
20 & 1.582843 & 1.582653 & 0.012 & 1.622204 & -2.49 \\
30 & 1.598142 & 1.597176 & 0.0605 & 1.698233 & -6.26 \\
40 & 1.620026 & 1.616958 & 0.189 & 1.832495 & -13.1 \\
50 & 1.648995 & 1.641458 & 0.457 & 2.065975 & -25.3 \\
60 & 1.685750 & 1.669998 & 0.934 & 2.470172 & -46.5 \\
70 & 1.731245 & 1.701771 & 1.7 & 3.161204 & -82.6 \\
80 & 1.786769 & 1.735863 & 2.85 & 4.317044 & -142 \\
90 & 1.854075 & 1.771271 & 4.47 & 6.197893 & -234 \\
100 & 1.935581 & 1.806932 & 6.65 & 9.169686 & -374 \\
110 & 2.034715 & 1.841752 & 9.48 & 13.730732 & -575 \\
\hline
\end{tabular}

TABLE 1. Numerical comparison for $\hat{T}$.

The PM method provides, in general, better approximations for small perturbation parameters when it includes the most number of terms from (3.2). Even so, it is common that the obtained approximations are appropriate for a small interval of the domain of the approximate solution. Fig. 3 represents, graphically, the reason why this method is efficient for the case of the elliptic integrals represented by (5.2). It can be seen that the range of values for $E(\epsilon)$ is $(1, \pi / 2]$, that is, $1<E(\epsilon) \leq 1.57$. The extreme values of that range are given by points $A$ and $B$ in Fig. 3 (where $\lim _{\epsilon \rightarrow 1} E(\epsilon)=1$ and $\left.E(0)=1.57\right)$. Notice that the range for the incomplete elliptical integral is even lower. For instance, points $C$ and $D$ represent the "extreme" values for $E(\epsilon, 1): \lim _{\epsilon \rightarrow 1} E(\epsilon, 1)=0.8414$ and $E(0,1)=1$.

Under these conditions it seems reasonable that just a few terms are required from (5.5) to obtain a good approximation of (5.2); because, since the beginning, the first approximation $v_{0}(\phi)$ (see (5.10)) is "close" to its final value $E(\epsilon, \phi)$. For instance, in the case of complete integrals, at the limit $\epsilon \rightarrow 1$, the first approximation provides the value $v_{0}(\pi / 2)=1.57$, not far of the true value $\lim _{\epsilon \rightarrow 1} E(\epsilon)=1$. Moreover, for $E(\epsilon, 1)$, the $\lim _{\epsilon \rightarrow 1} E(\epsilon, 1)=0.8414$ and $E(0,1)=1$ are even closer. Our results have an accuracy similar to other approximations reported in the literature [2].

\subsection{Complete elliptic integral of the first kind and the nonlinear pendulum}

Unlike what happens with the elliptic integrals depicted by differential equation (5.2), the complete elliptic integral of the first kind is not appropriately represented by (5.18). From Table 1 it can be noticed that normalized version of (7.3) is a proper 
approximation for $\hat{T}$ only for angles within the $30^{\circ}$ range, possessing a relative percentage error lower than six percent. From the same table can be seen that for angles of $80^{\circ}$ the relative error is about $-142 \%$, and for angles of $110^{\circ}$ the relative error is about $-575 \%$. Therefore, the approximation given by (7.3) is, in general, totally inadequate. Instead of adding terms to (5.18), Section 6 explained a technique that coupled the Padé transformation and the PM method giving as result an approximate value for $K(\epsilon)$ by (6.1) (see (7.4)).

[12] used a coupling of HPM, Laplace transformation, He's polynomials, and Padé technology in order to obtain series solution of nonlinear exponential boundary layer equation. Unlike the aforementioned method, this work proposes the use of PM and Laplace-Padé methods to deal with the truncated power series.

A noticeable difference can be seen in Table 1 for the percentage of the relative error at $20^{\circ}$, it is just $0.012 \%$; for $80^{\circ}$ we obtain an error that is about $2.85 \%$. Thus, even for $110^{\circ}$, the obtained relative percentage error is $9.48 \%$. Therefore, the combination of both techniques is not only novel but accurate. Finally note the simplicity of (6.1) compared to the infinite series in (5.18).

\section{Conclusions}

This work shows that some nonlinear problems can be adequately approximated by using the PM method, even for large values of the perturbation parameter; as it was done for the elliptic integrals described by (5.2). In fact the first approximation (5.10) is close to the true value for $E(\eta, \phi)(5.15)$, for every $\eta \in[0,1]$ and $\phi \in[0, \pi / 2]$ (see Fig. 1 and Fig. 3). This contributes to the success of the method in this case and it could be useful for similar cases, so our method can replace other sophisticated and difficult methods. Section 6 showed that even though the method is not adequate, as it can be seen for integral (2.2), it is possible to use a novel technique that coupled the PM method and the Padé transformation to improve the approximation (as it was proved for (6.1)). This result is much more accurate than (5.18), as it can be seen in Table 1. Besides, it is handy and useful for engineering applications.

\section{REFERENCES}

[1] G. Adomian, "A review of the decomposition method in applied mathematics," J. Math. Anal. Appl., vol. 135, no. 2, pp. 501-544, 1988.

[2] G. B. Arfken and H. J. Weber, Mathematical methods for physicists. 6th ed. Amsterdam: Elsevier/Academic Press, 2005.

[3] L. M. B. Assas, "Approximate solutions for the generalized KdV-Burgers equation by He's variational iteration method," Phys. Scr., vol. 76, no. 2, pp. 161-164, 2007.

[4] E. Babolian and J. Biazar, "On the order of convergence of Adomian method," Appl. Math. Comput., vol. 130, no. 2-3, pp. 383-387, 2002.

[5] G. A. Baker, Essentials of Padé approximants. New York-San Francisco-London: Academic Press, a subsidiary of Harcourt Brace Jovanovich, Publishers, 1975. 
[6] A. Beléndez, C. Pascual, M. L. Álvarez, D. I. Méndez, M. S. Yebra, and A. Hernández, "Higher order analytical approximate solutions to the nonlinear pendulum by he's homotopy method," Phys. Scr., vol. 79, no. 1, p. 8, 2009.

[7] T. L. Chow, Classical Mechanics. John Wiley \& Sons Inc., 1995.

[8] M. El-Shaed, "Application of He's homotopy perturbation method to Volterra's integro-differential equation," Int. J. Nonlin. Sci. Num., vol. 6, pp. 151-162, 2005.

[9] D. J. Evans and K. R. Raslan, "The tanh function method for solving some important non-linear partial differential equations," Int. J. Comput. Math., vol. 82, no. 7, pp. 897-905, 2005.

[10] A. Fereidoon, Y. Rostamiyan, M. Akbarzade, and D. Ganji, "Application of He's homotopy perturbation method to nonlinear shock damper dynamics," Arch. Appl. Mech., vol. 80, pp. 641-649, 2010.

[11] U. Filobello-Nino, H. Vazquez-Leal, R. Castaneda-Sheissa, A. Yildirim, L. Hernandez-Martinez, D. Pereyra-Diaz, P.-S. A., and C. Hoyos-Reyes, "An approximate solution of Blasius equation by using HPM method," Asian J. Math. Stat., vol. 5, pp. 50-59, 2012.

[12] M. A. Gondal and M. Khan, "Homotopy perturbation method for nonlinear exponential boundary layer equation using Laplace transformation, He's polynomials and Padé technology," Int. J. Nonlin. Sci. Num., vol. 11, pp. 1145-1253, 2010.

[13] J.-H. He, "Homotopy perturbation technique," Comput. Methods Appl. Mech. Eng., vol. 178, no. 3-4, pp. 257-262, 1999.

[14] J.-H. He, "A coupling method of a homotopy technique and a perturbation technique for nonlinear problems," Int. J. Non-Linear Mech., vol. 35, no. 1, pp. 37-43, 2000.

[15] J.-H. He, "Homotopy perturbation method for solving boundary value problems," Phys. Lett., A, vol. 350, no. 1-2, pp. 87-88, 2006.

[16] J.-H. He, "Some asymptotic methods for strongly nonlinear equations," Int. J. Mod. Phys. B, vol. 20, no. 10, pp. 1141-1199, 2006.

[17] J.-H. He, "Variational approach for nonlinear oscillators," Chaos Solitons Fractals, vol. 34, no. 5, pp. 1430-1439, 2007.

[18] J.-H. He, "Recent development of the homotopy perturbation method," Topol. Methods Nonlinear Anal., vol. 31, no. 2, pp. 205-209, 2008.

[19] M. H. Holmes, Introduction to perturbation methods, ser. Texts in Applied Mathematics. New York: Springer-Verlag, 1995, vol. 20.

[20] Y. Khan and Q. Wu, "Homotopy perturbation transform method for nonlinear equations using He's polynomials," Comput. Math. Appl., vol. 61, no. 8, pp. 1963-1967, 2011.

[21] M. Merdan, A. Gökdoğan, and A. Yildirim, "On the numerical solution of the model for HIV infection of $C D 4^{+}$T cells," Comput. Math. Appl., vol. 62, no. 1, pp. 118-123, 2011.

[22] H. Vazquez-Leal, R. Castaneda-Sheissa, U. Filobello-Nino, A. Sarmiento-Reyes, and J. J. Sanchez-Orea, "High accurate simple approximation of normal distribution integral," Math. Probl. Eng., vol. 2012, p. 22, 2012.

[23] H. Vazquez-Leal, U. Filobello-Nino, R. Castaneda-Sheissa, L. Hernandez-Martinez, and A. Sarmiento-Reyes, "Modified HPM methods inspired by homotopy continuation methods," Math. Probl. Eng., vol. 2012, p. 19, 2012.

[24] F. Xu, "A generalized soliton solution of the Konopelchenko-Dubrovsky equation using He's expfunction method," Z. Nat.forsch., A: Phys. Sci., vol. 62, no. 12, pp. 685-688, 2007.

[25] L.-N. Zhang and L. Xu, "Determination of the limit cycle by He's parameter-expansion for oscillators in a $u^{3} /\left(1+u^{2}\right)$ potential," Z. Nat.forsch., A: Phys. Sci., vol. 62, no. 7-8, pp. 396-398, 2007. 


\section{Authors' addresses}

\section{U. Filobello-Nino}

University of Veracruz, Department of Electronic Instrumentation, Cto. Gonzalo Aguirre Beltan S/N, 91000 Xalapa, Veracruz, Mexico

E-mail address: ufilobello@uv.mx

\section{H. Vazquez-Leal}

University of Veracruz, Department of Electronic Instrumentation, Cto. Gonzalo Aguirre Beltan S/N, 91000 Xalapa, Veracruz, Mexico

E-mail address: hvazquez@uv.mx

\section{Y. Khan}

Zhejiang University, Department of Mathematics, Hangzhou 310027, China

E-mail address: yasirmath@yahoo.com

\section{A. Yildirim}

Ege University, Department of Mathematics, 35100 Bornova, İzmir, Turkey

Current address: University of South Florida, Department of Mathematics and Statistics, 336205700, Tampa, FL., USA

E-mail address: ahmet.yildirimeemail.net

\section{M. Jimenez-Fernandez}

University of Veracruz, Department of Electronic Instrumentation, Cto. Gonzalo Aguirre Beltan S/N, 91000 Xalapa, Veracruz, Mexico

E-mail address: vicjimenez@uv.mx

\section{A. L. Herrera-May}

University of Veracruz, Micro and Nano Technology Research Center, Boca del Rio, Veracruz, Mexico

E-mail address: leherrera@uv.mx

\section{R. Castaneda-Sheissa}

University of Veracruz, Department of Electronic Instrumentation, Cto. Gonzalo Aguirre Beltan S/N, 91000 Xalapa, Veracruz, Mexico

E-mail address: rocastaneda@uv.mx

\section{J. Cervantes-Perez}

University of Veracruz, Department of Electronic Instrumentation, Cto. Gonzalo Aguirre Beltan S/N, 91000 Xalapa, Veracruz, Mexico

E-mail address: jcervantes@uv.mx 